Brit. J. vener. Dis. (1962), 38, 171.

\title{
TWO INTERNATIONAL MEETINGS IN WASHINGTON, D.C.
}

\begin{abstract}
The World Forum on Syphilis and Other Treponematoses and the XII International Congress of Dermatology were held in Washington, D.C. during the first and second weeks of September, 1962. Both meetings attracted a large international gathering and took place in two adjacent, gigantic, congress hotels in beautiful surroundings and in well-designed, attractive conference rooms. A great deal of hard work and attention to detail had been put into the preliminary preparations of both meetings and the programmes were extensive, ambitious, and comprehensive.
\end{abstract}

\section{WORLD FORUM ON SYPHILIS}

The World Forum on Syphilis and Other Treponematoses was sponsored by the American Social Health Association, the American Venereal Disease Association, and the U.S. Public Health Services. Other participating agencies were the World Health Organization and the International Union against the Venereal Diseases and the Treponematoses. The Forum was inspired by the world-wide increase in syphilis and the persistence of other treponemal diseases in many areas.

The organization and planning committee concerned itself with the clinical, public health, and social and behavioural aspects of the problem. The programme consisted of morning Plenary Sessions, which were composed of authoritative papers by experts in various fields, reviewing different aspects of the problems of treponemal diseases. These included discussions on international cooperation, population mobility in the control of syphilis, the present position of medical research, epidemiology, clinical and laboratory aspects of diagnosis, the social aspects of venereal diseases, and the role of the behavioural sciences in venereal disease programmes.
The afternoon sessions were divided into three sections. A medical section heard papers principally concerned with clinical problems, a public health section considered means of spread and methods of control, and a behavioural science section discussed the problems of human behaviour and their relationship to the venereal diseases.

The opening address was given by Luther L. Terry, Surgeon General, United States Public Health Service. This was followed by a paper from Thorstein Guthe of the World Health Organization which outlined the scope of the problem of treponemal diseases in the world today. William J. Brown of the United States Public Health Service set the tone of the meeting in a paper putting forward the concept of total eradication of syphilis and other treponemal diseases. $\mathrm{He}$ argued that the increase in incidence of infectious syphilis since 1957 suggested that control of the disease, even with effective modern methods, was unlikely to be successful. He described his Public Health Law of Diminishing Returns, which stated that "when a disease eradication campaign reaches near success it is more likely to be the campaign 
which disappears than the disease". 20,000 cases of syphilis were reported in the U.S.A. during 1961 and 4,000 people died of the disease. "What would have been the reaction of the public if the same numbers had contracted smallpox, typhus, or other infectious diseases?", Brown asked.

The value of "cluster" contact tracing was stressed by several speakers. Briefly this consists of examining and testing not only the sexual contacts of patients with infectious syphilis but also their immediate friends and associates and those who have the same social habits and behaviour. It was claimed that "cluster" testing had led to a 20 per cent. increase in case-finding since it had been employed on a wide scale in the United States. Others indicated the importance of re-interviewing patients about contacts on their second and subsequent visits; one speaker stated that, of every 100 patients re-interviewed, forty named additional contacts at a subsequent interview. Speed in obtaining particulars of contacts was also very important.

In the clinical section, I. Schamberg expressed the opinion that the decrease in the incidence of syphilis from 1949 to 1955 was principally due to the wide use of penicillin in the community for non-syphilitic disease. Owing to the increase in the number of sensitivity reactions to penicillin and to the ease of administration, the broad-spectrum antibiotics have been more widely used since 1955 . These drugs have less antisyphilitic effect and it was predicted that the increase in infectious syphilis was likely to continue because penicillin will never again be used on such a wide scale.

Several other papers stressed the increasing number of patients who were sensitive to penicillin and pointed out that an "anti-anaphylaxis kit" should always be available. A detailed review of the effect of drug sensitivity on treponemal control programmes was given by R. R. Willcox. A new method of testing for penicillin sensitivity was described and was stated to have given promising preliminary results. The use of other antibiotics in the treatment of treponemal disease was described by several observers. Experience was still very limited and schedules of dosage varied widely.

The treatment of 8 th nerve deafness in congenital syphilis and certain other conditions using large doses of prednisone was described by R. D. Hahn. Progress was assessed by audiometry, and definite improvement had been observed in over 50 per cent. of cases treated. Speech discrimination was one of the most difficult problems and sometimes remained unaffected by therapy.

The persistence of Treponema pallidum in the inguinal lymphatic nodes of experimental animals and in man in late syphilis, despite treatment, was reported by $P$. Collart. Special staining techniques had been used and $T$. pallidum was demonstrated in the inguinal nodes of rabbits 8 to 12 months after treatment. The organism had also been demonstrated in twelve cases of syphilis of over 2 years' duration.

Serological tests and immunological aspects of the treponematoses were reviewed by several speakers. Recent advances in the Fluorescent Treponemal Antibody Test show some progress with techniques but the test does not appear to be simple or reliable enough for routine use at the present time. New developments in rapid serological testing were discussed by J. Portnoy. He described the Rapid Plasma Reaction (RPR) Card Test, a flocculation test performed on a specially-prepared card using an antigen containing carbon. The test can be performed in less than 10 minutes and the equipment required is simple and disposable. The test had been compared with the V.D.R.L. test on a large number of sera and agreement between the tests was reported to be good. This technique may have certain advantages for field work but much remains to be done to assess its specificity, sensitivity, and reproducibility before it can be used for the clinical assessment of patients.

The section concerned with the behavioural sciences was a new departure in conferences concerned with syphilis and treponemal diseases and many original and interesting papers were presented. Discussion of the patients' attitudes and reactions to venereal diseases and to education and information about them, and of the reaction of doctors to patients with syphilis produced some stimulating and valuable ideas. Social workers, psychiatrists, physicians, nurses, and health visitors all contributed to this section. The importance of educating the public about the problems of infectious venereal diseases was stressed and methods of instructing young people were discussed. C. S. Nicol considered the attitude of the public towards venereal diseases and suggested that young people to-day were subjected to the worst influences of peacetime and also, owing to the everpresent menace of the bomb, to the worst influences that prevail in war and periods of uncertainty. C. S. Deschin analysed the prevailing standards and values of youth in a detailed paper and $\mathrm{C}$. Winick discussed 
the contribution that the behavioural sciences could make in helping to control or eradicate venereal diseases.

The planning and organization of the Forum were of the highest quality and the programme was carried out smoothly and efficiently. Although little in the way of new facts may have emerged, the Forum was a stocktaking of current knowledge and a restatement of the present position regarding the treponemal diseases. All those who attended it must have been stimulated to review their own methods and attitudes to this important group of diseases.

\section{INTERNATIONAL CONGRESS OF DERMATOLOGY}

The XII International Congress of Dermatology was held the following week in an adjacent hotel. There were over 3,000 active participants and the programme was very varied. A whole day was devoted to the treponemal diseases and non-treponemal venereal diseases including gonorrhoea. The Congress, under the presidency of Donald M. Pillsbury, was well planned, but the large number of delegates caused considerable congestion in the main conference rooms and at the various information centres.

The highlight of the Congress was certainly the coloured television programme in which cases of psoriasis were presented in England and seen and discussed by dermatologists at the Congress in Washington using the Telstar earth satellite. The fact that pictures of skin diseases in colour can be successfully transmitted more than 3,000 miles and that physicians can discuss diagnosis and treatment of patients between two distant centres simultaneously is a measure of the breathtaking progress which has occurred in this aspect of technology and science during the past decade.

There was some slight repetition in the papers on syphilis and the treponemal diseases from the World Forum the previous week. However, E. Gurney Clark discussed the natural course of untreated syphilis and S. Olansky redescribed the results of experimentally-induced syphilis in man. G. d'Alessandro gave an interesting account of the antigenic structure of Treponema pallidum. A. H. Wheeler reported on immunity in experimental syphilis.

In the afternoon session, C. Grupper reported further experience with cortisone and its derivatives in the prevention of Herxheimer reactions during treatment of syphilis. J. M. Knox discussed alternative drugs in the treatment of syphilis. W. G. Simpson presented a paper on penicillin reactions. The management of trichomoniasis was discussed by both P. Durel and R. D. Catterall; oral metronidazole was agreed to be the treatment of choice in this condition toxic effects having so far been few and of minor importance. W. E. Deacon described the fluorescent antibody identification of Neisseria gonorrhoeae. Trends in in vitro testing of $N$. gonorrhoeae to antibiotics was reported by A. Reyn. With simplification of the techniques, fluorescent antibody tests may have an important place in the diagnosis of gonorrhoea, especially in women. Increases in the numbers of strains of gonococci which are relatively insensitive to penicillin were reported and confirmed by other speakers. Finally, R. R. Willcox reviewed the treatment of gonorrhoea. It was agreed that penicillin remained the drug of choice and that it should be given in adequate dosage.

Several papers presented during the remainder of the congress were important to venereologists with an interest in general medicine. The symposium on clinical assays and new experimental methods contained much that was of general application; a communication by V. Wright on psoriatic arthritis was very helpful to those interested in Reiter's disease; discussions on virus-induced tumours of the skin revealed new information about Molluscum contagiosum and warts.

The large section devoted to scientific exhibits contained amongst others an excellent demonstration of the dental changes in congenital syphilis by T. Putkonen, Behçet's syndrome by H. O. Curth, and annotated photographs of conditions resembling leprosy by P. Fasal. Clinical cases of skin diseases were presented at the Walter Reed General Hospital, Washington, the University of Maryland Hospital, Baltimore, and the Johns Hopkins Hospital, Baltimore.

In all international conferences there is an optimum size. If the number of delegates is too great the situation becomes unmanageable by a single 
organisation. The officers and committees of the XII International Congress of Dermatology are to be congratulated on their valiant efforts in dealing so effectively with the large number of members who almost overflowed the available accommodation. Another problem at such meetings is the very varied standards of the papers presented. Careful selection should ensure that a minimum standard is achieved and that papers containing new material are not excluded because of lack of space on the programme. On the other hand, papers containing no new material which are given for prestige reasons only, should be reduced to a minimum or eliminated altogether.

The World Forum on Syphilis and the International Congress of Dermatology brought together experts and specialists from all over the world. Personal exchanges of ideas and information and the formation of new friendships are amongst the most important results of such gatherings. The meetings in Washington have contributed significantly to the understanding of many different medical problems, and have served to bring doctors of all nationalities closer together and to raise the standards of medicine throughout the world.

\section{REVISED SUBSCRIPTION RATES}

The attention of subscribers is drawn to the notice on the back cover regarding increased prices for B.M.A. periodicals. The rate to members of the M.S.S.V.D. will not be affected as their subscription to the Society includes the cost of the British Journal of Venereal Diseases. 